\title{
Programmable nucleic acid nanoswitches for the rapid, single-step detection of antibodies in bodily fluids
}

\author{
Alessandro Porchetta, ${ }^{1, \#}$ Rudy Ippodrino, ${ }^{2, \#}$ Bruna Marini, ${ }^{2}$ Arnaldo Caruso, ${ }^{3}$ Francesca Caccuri ${ }^{3}$ \\ and Francesco Ricci ${ }^{1, *}$ \\ ${ }^{1}$ Department of Chemistry, University of Rome, Tor Vergata, Via della Ricerca Scientifica, oo133, Rome, Italy. \\ ${ }^{2}$ Ulisse BioMed S.r.l., Area Science Park, 34149, Trieste, Italy. \\ ${ }^{3}$ University of Brescia Medical School, Dept. of Molecular and Translational Medicine, Section of Microbiology, \\ Piazzale Spedali Civili 1, 25123 Brescia, Italy.
}

\begin{abstract}
Antibody detection plays a pivotal role in the diagnosis of pathogens and monitoring the success of vaccine immunization. However, current serology techniques require multiple, time-consuming washing and incubation steps, which limit their applicability in point-of-care (POC) diagnostics and high-throughput assays. We developed here a nucleic acid nanoswitch platform able to instantaneously measure Immunoglobulins of type G and E (IgG and IgE) levels directly in blood serum and other bodily fluids. The system couples the advantages of target-binding induced colocalization and nucleic acid conformational-change nanoswitches. Due to the modular nature of the recognition platform the method can potentially be applied to the detection of any antibody for which an antigen can be conjugated to a nucleic acid strand. In this work we show the sensitive, fast and cost-effective detection of four different antibodies and demonstrate the possible use of this approach for the monitoring of antibody levels in HIV+ patients immunized with AT2o therapeutic vaccine.
\end{abstract}

\section{INTRODUCTION}

Antibody detection is crucial for the diagnosis of many human disorders including infectious, autoimmune, and oncological diseases. ${ }^{1,2}$ Nowadays serological tests for early diagnosis require not only to be sensitive, quantitative and specific but also rapid and easy to use. Unfortunately, however, current methods routinely used for this purpose in clinical settings often require reagentintensive laboratory-based techniques (e.g ELISA, Western Blot assay, radioimmunoassay). In an attempt to find new solutions to this problem several approaches for the fast and sensitive detection of antibodies have been proposed. For example, the most common approach in this rapidly developing area has been to miniaturize stateof-the-art analytical work flows such as immunoassays using microfluidics and lab-on-chip technology. ${ }^{3-5}$ Other methods, instead, take advantage of the observation that IgG and IgE antibodies have two identical binding sites separated by about 10-12 $\mathrm{nm}$. Such spatial geometry, that is shared by all $\operatorname{IgG}$ and $\operatorname{IgE}$ antibodies (i.e. most diagnostically relevant antibodies), has been cleverly used to design and develop novel methods for antibodies detection that are rapid, sensitive and cost-effective.

In this regard several examples have been demonstrated in which a pair of antigen-conjugated signalling probes, upon binding to the two binding sites of the same target antibody, are co-localized in a confined volume. This, in turn, dramatically increases the local concentration of the reporter probes and activates their signalling function. ${ }^{6,7}$ For example, Heyduk and coworkers have used FRET-labelled DNA probes that are able to hybridize and generate a FRET signal only when bound to the target antibody. ${ }^{8,9}$ Potential drawbacks of the latter strategy include limited signal gains (due to the high background of the free fluorophore in absence of antibody) and non-trivial design issues of the sensing elements. Co-localization of ligands on one antibody has been also employed to allow antibody-induced reconstitution of enzyme activity. ${ }^{10}$ However, this approach often fails in reconstituting full enzyme activity thus affecting the sensitivity of the method. To overcome this problem, Merkx and coworkers ${ }^{11}$ introduced antibody-based disruption of an enzyme-inhibitor complex; although this system displayed increased sensitivity, the reporter enzyme showed attenuated activity in serum which might limit its use with real samples.

Recently, we and others have employed antigenconjugated switching probes rationally designed to undergo a conformational change upon binding to a specific target antibody that ultimately leads to a change in signal (either optical or electrochemical). ${ }^{12-18}$ In one of these formats we have designed a DNA nanoswitch conjugated with two identical antigens and a fluorophore/quencher signalling pair. ${ }^{19}$ When the target antibody binds to each of the two antigens, a conformational change occurs leading to an increase in fluorescence signal. In order to function properly, however, a careful adjustment of the distance between the two identical antigens is critical but difficult to achieve especially for larger antigens.

In order to improve upon the above approaches we show here the rational design of programmable nucleic acid nanoswitches for the rapid, single-step detection of antibodies in bodily fluids. The approach we propose couples the advantageous features of DNA-based conformational switching probes (nanoswitches) with those of co-localization based methods but allows to 
overcome the practical limitations affecting the above described platforms.

\section{RESULTS AND DISCUSSION}

Sensing principle of programmable DNA-based nanoswitches. Our strategy to achieve a programmable nucleic acid nanoswitch for the rapid, single-step detection of antibodies is based on the use of a set of three DNA-based elements that are rationally designed to give a measurable signal in the presence of a specific target antibody. The first element (strand \#1, Figure 1, left) is a synthetic DNA sequence designed with two selfcomplementary portions that lead to a stem-loop conformation flanked by a 15 -base DNA tail. Strand \#1 is also modified at the two ends of the stem portion with a fluorophore/quencher pair responsible for signalling. The second element (strand \#2, Figure 1, left) is a synthetic DNA strand that is conjugated to one copy of an antigen (i.e. the recognition element indicated by the green hexagon in Figure 1, left) and contains a 15-base sequence (orange portion in Figure 1, left) complementary to the tail of strand \#1 which allows efficient coupling with it. The third element (strand \#3, input strand, Figure 1, left) is conjugated with another copy of the antigen and contains a domain complementary to the loop sequence of strand \#1 (blue portion) flanked by a 15-base poly-T linker portion. Of note, strands \#1 and \#3 are designed so that their binding affinity, in the absence of the target antibody, is poor. Binding of the antibody to the antigens of strand \#2 and \#3, brings the loop of strand \#1 and its cognate portion of strand \# 3 into close proximity thereby dramatically increasing their local concentrations (Figure 1, right). This allows efficient binding between strand \#1 and strand \#3 leading to an increase of the observed fluorescence signal.

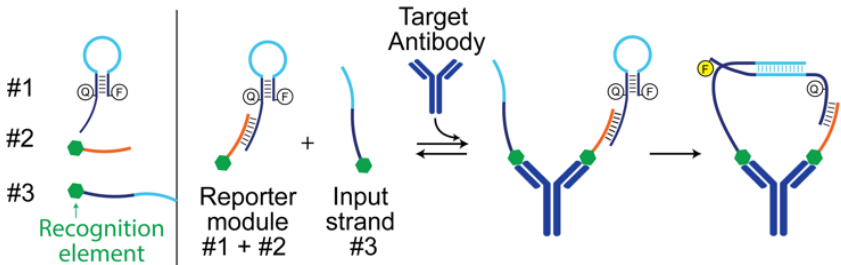

Figure 1. Programmable nucleic acid nanoswitches activated by antibody induced co-localization. The nanoswitch is composed of three synthetic nucleic acid strands. A first DNA strand (strand \#1) is a fluorophore/quenchermodified DNA stem-loop flanked by a 15-base single-stranded tail. To create an antibody-reporter module, this tail is hybridized with a second synthetic DNA strand (strand \#2) conjugated to an appropriate recognition element (i.e. an antigen, green hexagons). The third element (strand \#3, input strand) is a DNA strand conjugated with another copy of the recognition element and contains a portion complementary to the loop sequence of strand \#1. In the presence of the target antibody the reporter module (strand $\#_{1}+$ strand $\#_{2}$ ) and the input strand (strand \#3) are co-localized in a confined volume. This brings the stem-loop of strand $\#_{1}$ and the input strand $\#_{3}$ into close proximity, thereby increasing their local concentrations and allowing their efficient hybridization.
Programmable DNA-based nanoswitches for AntiDNP antibodies detection. In order to achieve proof of principle of the method, we initially used the small molecule 2,3-dinitrophenol (DNP) as recognition element (i.e. antigen) and an anti-DNP IgE antibody as the target analyte (Figure 2a). We designed strand \#1 with a highly stable 5-base stem containing $4 \mathrm{GC}$ and 1 AT base pairs and a 15-base loop. Such DNA stem-loop structure is stable from 25 to $35{ }^{\circ} \mathrm{C}$, making the system suitable for clinical applications (Figure SI1). This design prevents any significant unwanted annealing reactions with strand \#3 in the absence of the target antibody. To achieve optimal sensitivity for antibody detection, we tested different lengths of the complementary domain of strand \# 3 to the strand \#1 loop (ranging from 13 to 17 bases). As expected, binding curves performed at a fixed equimolar concentration of strand \#1 and \#2 (leading to the reporter module, see Figure 1) combined with increasing concentrations of strand \#3 (input strand) showed different affinities with a length-dependent fashion (Figure SI2). In the presence of a saturating concentration of the target antibody (i.e. $100 \mathrm{nM}$ ) we observed an improvement of the binding affinity with all the tested sequences (Figure $2 \mathrm{~b}$ and Figure $\mathrm{SI}_{3}$ ). The lengths of complementary portion of strand \#3 that lead to the largest change in affinity are 15 and 16 bases (Figure 2c). We selected for further experiments the 15-base long strand \#3 to prevent any spurious unwanted hybridization due to minimal changes of the experimental conditions (i.e. ionic strength, $\mathrm{pH}$, etc) which might occur when using real samples. Using this input strand we observed a concentration-dependent change of binding affinity between the DNP-reporter module and DNP-input strand induced by the presence of the anti-DNP antibody (Figure 2d).
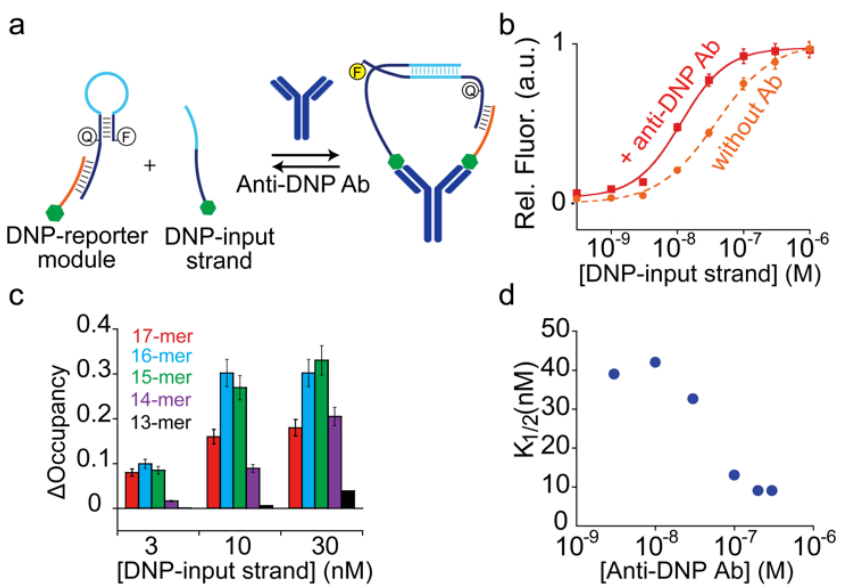

Figure 2. Programmable DNA-based nanoswitches for AntiDNP antibody detection. a) As proof-of-principle model we used 2,3-dinitrophenol (DNP) as the recognition element and anti-DNP as target antibody. b) Binding affinity testing between DNP-reporter module (strand \#1 + strand \#2, $10 \mathrm{nM}$ ) and its complementary 15-base DNA-input strand (strand \#3) in the absence (orange) and in the presence (red) of a saturating concentration of anti-DNP antibody (i.e. $100 \mathrm{nM}$ ). c) Antibody- 
induced difference in occupancy at three different concentrations (i.e. 3,10 and $30 \mathrm{nM}$ ) of strand \#3 of different lengths (i.e. from 13 to 17 bases) and at fixed concentrations of reporter module (strands $\#_{1}+\# 2,10 \mathrm{nM}$ ) in the presence and absence of Anti-DNP antibody (100 nM). d) $K_{1 / 2}$ (input strand concentration at which we observe half the maximum signal change) values for the binding between the DNP-reporter module (10 $\mathrm{nM})$ and the DNP-input strand as a function of a fixed concentration of anti-DNP antibody in solution. Each value is the average of three determinations \pm the standard deviation. The experiments have been performed at $35^{\circ} \mathrm{C}$, in a $100 \mu \mathrm{L}$ solution of $\mathrm{Na}_{2} \mathrm{HPO}_{4}(50 \mathrm{mM})$ and $\mathrm{NaCl}(150 \mathrm{mM})$ at $\mathrm{pH}$ 7.0 with the DNP-reporter module, input strand and anti-DNP antibody at the indicated concentrations.

The above characterization allowed us to select the optimal concentration of reporter module (i.e. $10 \mathrm{nM}$ ) and input strand (i.e. 10 $\mathrm{nM}$ ) for antibody detection. Under such experimental conditions our platform responds rapidly to anti-DNP antibodies generating a 300 -fold increase in fluorescence at saturating concentration of anti-DNP antibodies within two minutes (Figure za) and achieving sensitivities in the low nanomolar range $\left(\mathrm{K}_{1 / 2}=\right.$ $8 \pm 4 \mathrm{nM}$; Figure 3b). Notably, because the signal is due to the binding of the antibody to the two recognition elements the platform is highly specific and no significant signal change occurs in the presence of non-specific antibodies (i.e. $100 \mathrm{nM}$ ) (Figure 3c). The platform can be also deployed to detect free antigen (i.e. DNP) in solution in a single-step competitive homogeneous format (Figure SI4). Control experiments carried out in the absence of strand \#2 or strand \#3 further support the proposed sensing mechanism (Figure $\mathrm{SI}_{5}$ and SI6). Experiments with different lengths (poly-T of 3 and 40 bases) of the linker portion of strand \#3 (see Figure 1 ) serve as another supports to our sensing principle mechanism. A very short linker ( 3 bases) does not allow efficient binding of the input strand to the reporter module, likely due to the fact that its length is insufficient to cover the $12 \mathrm{~nm}$ distance between the two antibody binding sites (Figure SI7). Similarly, a much longer linker (40 bases) while providing sufficient length also provides a higher conformational freedom that ultimately affects the colocalization effect and thus signalling (Figure $\mathrm{SI}_{7}$ ).

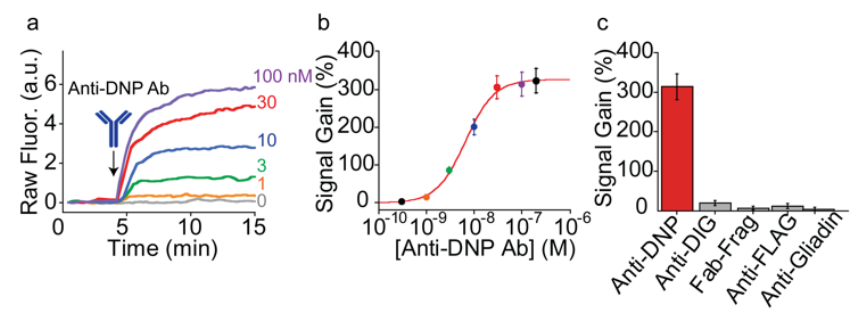

Figure 3. Anti-DNP antibody detection based on DNA nanoswitch activation triggered by co-localization. a) Kinetic experiments performed through the addition of different concentrations of anti-DNP antibody in solution. b) Signal gain as a function of antibody concentration $\left(K_{1 / 2}=8 \pm 4 \mathrm{nM}\right)$. c) Determination of the nanoswitch specificity comparing target with negative controls (i.e. $100 \mathrm{nM}$ ). The experiments here were conducted at $35^{\circ} \mathrm{C}$ in a $100 \mu \mathrm{L}$ solution of $\mathrm{Na}_{2} \mathrm{HPO}_{4}(50 \mathrm{mM})$ and $\mathrm{NaCl}(150 \mathrm{mM})$ at $\mathrm{pH} 7.0$ containing the DNP-reporter module
(10 $\mathrm{nM}$ ) and the input strand (10 $\mathrm{nM}$ ) and by adding increasing concentration of anti-DNP antibody.

One of the major advantages of our sensing mechanism is that it is versatile: changing the recognition elements allows us to potentially detect any class of target antibodies and antigens. To demonstrate this we have employed a different recognition element (i.e. digoxigenin, DIG) and conjugated it to strand \#2 and \#3. With this new set of sequences we have measured antiDIG antibodies (IgG) reaching sensitivity and specificity similar to those observed for the anti-DNP antibody (Figure SI8). This demonstrates that the approach is flexible and versatile and, because all IgG and IgE antibodies share the same y-shape geometry that supports the proposed detection mechanism, potentially adaptable to the detection of other antibodies.

Modular programmable DNA nanoswitches for antibodies detection. To further demonstrate the possible utility of our platform for diagnostic applications we then decided to move to more complex antigens. Peptides are by far the most representative class of antigens for diagnostic antibodies. The attachment of peptides to DNA sequences is, however, expensive and challenging from a synthetic point of view, ${ }^{20-22}$ two factors that would affect the potential application of our platform for diagnostic use. Motivated by the above considerations we have thus designed a modular version of the above described platform that would still require a total of 3 oligonucleotides but where only one of them is labeled with the antigen. Such antigen-conjugated strand (strand \#2 in Figure 4a) acts as a scaffold to bind both the stemloop reporter strand and the input strand (i.e. strand \#3) (Figure $4 \mathrm{~b}$ ). In this case the antigen-conjugated strand is made of peptide-nucleic acid (PNA), a DNA mimic that, while still maintaining a high sequence specificity towards DNA complementary strands, has a pseudopeptide backbone which makes the conjugation with peptides easier. ${ }^{23}$ The modular nature of such approach renders it easily applicable to the detection of different antibodies through the simple expedient of changing only the strand \#2 (antigen-conjugated strand). With this new modular and versatile approach we have first employed as recognition element a 12-residue long HIV-1-p17 epitope (p17) recognizing the anti-HIV-1 p17 antibody (Figure $4 \mathrm{~b}$ ). This modular platform results to be equally sensitive, rapid and specific (Figure 4c) as the non-modular version shown above (Figure 2-3). The antiHIV-1 p17 targeting antibody sensors likewise exhibits similar signal gains (between $260 \%$ and $300 \%$ ), suggesting that the larger antigens employed here do not affect the interaction between the reporter module and the input module. The high specificity of the sensor and its high signal gain due to the binding-induced conformational change of the reporter module in the presence of the anti$\mathrm{HIV}_{1}$ p17 antibody, renders our sensors selective and sensitive enough to be deployed in complex samples. Our anti-HIV-1 p17 antibody sensor performs well when used in $90 \%$ blood serum, producing a $K_{1 / 2}$ value (i.e. $24 \pm 8$ 
nM) comparable to that obtained in phosphate buffer (i.e. $20 \pm 4 \mathrm{nM}$ ) (Figure SI9) and the platform does not show any sign of degradation in this matrix over the time scale of the experiments (Figure SI10). We note, however, that the gain observed in blood serum is significantly lower probably due to the higher background fluorescence of this sample matrix.

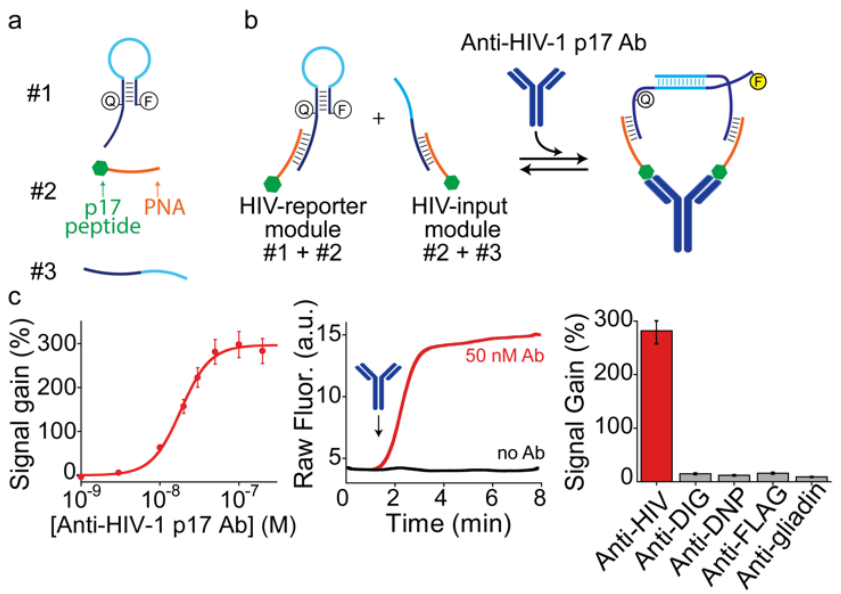

Figure 4. Modular sensing platform for the detection of anti-HIV-1 p17 antibodies. a) We used a PNA-peptide chimera (strand \#2) as the strand responsible for the binding to strand \#1 and strand $\#_{3}$. Such PNA-peptide chimera presents a specific HIV-1-p17 epitope (p17) as recognition element. b) The PNApeptide chimera allows the HIV-reporter module $\left(\#_{1}+\#_{2}\right)$ and the HIV-input module $(\# 2+\# 3)$ to be into close proximity only in the presence of the target antibodies against p17 epitope, leading to an increase of the observed fluorescence signal. c) The modular approach detects anti-HIV-1 p17 antibodies at low nanomolar concentrations $\left(\mathrm{K}_{1 / 2}=20 \pm 4 \mathrm{nM}\right.$, left); Fluorescence kinetics show that the detection is very rapid, achieving $90 \%$ of the fluorescence signal change after less than three minutes (middle); the modular sensor is highly specific and it does not exhibit any significant response in the presence of off-target antibodies (i.e. $100 \mathrm{nM}$, right). The experiments have been performed at $35^{\circ} \mathrm{C}$ in a $100 \mu \mathrm{L}$ solution of $\mathrm{Na}_{2} \mathrm{HPO}_{4}(50 \mathrm{mM})$ and $\mathrm{NaCl}(150 \mathrm{mM})$ at $\mathrm{pH} 7.0$ containing the HIV-reporter and input modules (strand \#1, $20 \mathrm{nM}$; strand \#2, $40 \mathrm{nM}$; strand \#3, $20 \mathrm{nM}$ ) at different concentrations of anti-HIV-1 p17 antibodies. Specificity test gives the signal gains obtained under the above experimental conditions and by adding saturating concentrations (i.e. $100 \mathrm{nM}$ ) of the anti-HIV-1 antibody and other non-specific antibodies (all IgG and IgE antibodies).

To further explore the potential of our platform we have tested the possibility of multiplex measurement using the same nanoswitches demonstrated above for the detection of different antibodies (i.e. Anti-DNP, Anti-DIG and Anti-p17 antibodies) and each labeled with a different fluorophore/quencher pairs having distinguishable wavelengths of fluorescence emission. The results demonstrate that we are able to simultaneously detect the three target antibodies in the same solution in an orthogonal way, with signal gains and response times that are not affected by the presence of the other switches (Figure 5).
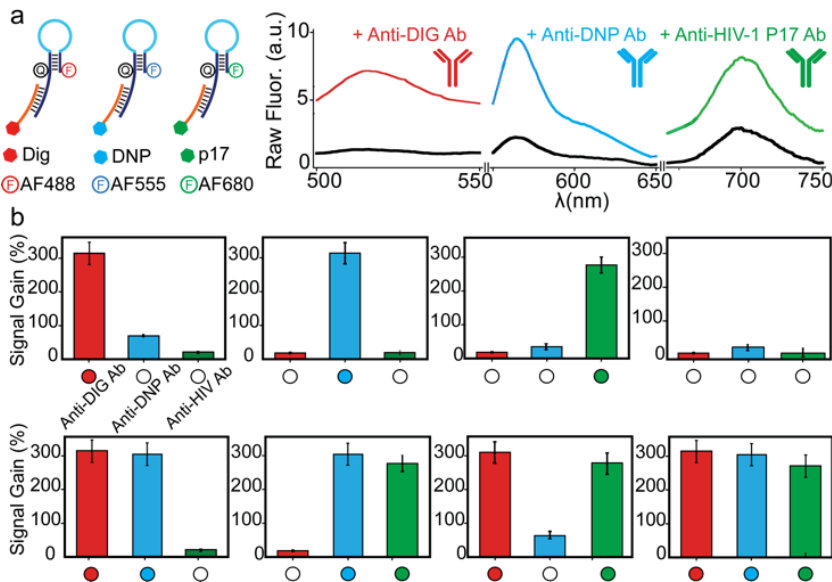

Figure 5. Orthogonal multiplex antibody detection using programmable DNA nanoswitches. a) Schematics of three reporter modules for the simultaneous multiplex detection of anti-DIG, anti-DNP and anti-HIV-1 p17 antibodies (left). Fluorescence emission spectra measurement in presence and absence of the three target antibodies (i.e. $50 \mathrm{nM}$ ). Each reporter module contains its specific input module that can be independently activated in the presence of its cognate antibody (right). b) Signal gain of the three nanoswitches obtained by adding each antibody in different combinations. Filled and blank circles are used to identify the antibody added in solution. Error bars represent the standard deviation from at least three replicates. The experiments were performed at $35^{\circ} \mathrm{C}$ in a $100 \mu \mathrm{L}$ solution of $\mathrm{Na}_{2} \mathrm{HPO}_{4}(5 \mathrm{O} \mathrm{mM})$ and $\mathrm{NaCl}(150 \mathrm{mM})$ at $\mathrm{pH} 7.0$ containing the three different nanoswitches (each with the same reporter module and input module/strand concentration reported in Figure 3-4).

Detection of Anti-AT2o antibodies in human blood plasma of vaccinated patients. A clinically relevant application of our platform is the monitoring of the immune response elicited from patients that are immunized with a peptide-based therapeutic HIV vaccine. Historically, successful vaccines have been prepared from live attenuated bacteria or viruses or inactivated viruses. In recent years, significant progress has been made to enable a more mechanistic approach to vaccine research and development compared with the traditional empirical approaches. ${ }^{24}$ The use of peptides as minimal pathogen components able to stimulate long lasting protection is becoming a pivotal strategy in vaccine development. ${ }^{25}$ In this context, peptide-based vaccination represents a promising approach to treat HIV-1 infected patients by boosting or redirecting immune system to neutralize critical HIV-1 antigens whose biological effects are relevant in the context of viral pathogenesis. ${ }^{26}$ On the basis of this evidence and successful preclinical data, ${ }^{27,28}$ a 20 amino acid synthetic peptide (AT20) representative of the p17 functional region, coupled to the carrier protein keyhole limpet hemocyanin $(\mathrm{KLH})$ was selected as the active agent to perform a therapeutic phase I clinical trial in highly active antiretroviral therapy (HAART)-treated HIV-1-infected patients. While during the natural course of $\mathrm{HIV}_{-1}$ infection the occurrence of an antibody response to AT2O is rare or at very low titer, ${ }^{29}$ it was demonstrated that all 
vaccinated patients developed high titers of high-avidity anti-AT2o antibodies. ${ }^{27}$

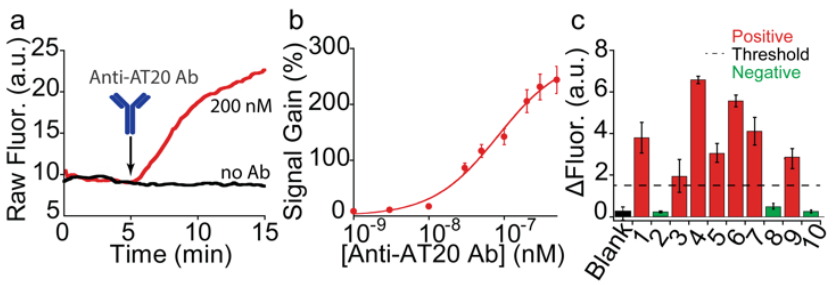

Figure 6. Detection of Anti-AT2o antibodies in human blood plasma from vaccinated individuals. a) Fluorescence kinetics experiments show that the detection of anti-AT2o antibodies is rapid. b) The modular sensor detects anti-AT2o antibodies at nanomolar concentrations in $10 \%$ human blood plasma $\left(\mathrm{K}_{1 / 2}=80 \pm 10 \mathrm{nM}\right)$; c) Anti-AT2o antibodies detection in $10 \%$ blood plasma samples of different HIV-positive patients (numbered from 1 to 10 ) as a way to monitor the immune response induced by the AT-2o vaccine treatment. Seven HIVpositive patients that received the AT2o-based HIV-vaccine were easily identified as positive (positive, orange); HIV-positive, nonvaccinated patients tested negative (negative, green). Three different blank blood plasmas were used as negative controls. Threshold value was calculated as the fluorescence signal corresponding to the mean fluorescence signal obtained with three different blank samples of HIV-negative patients +3 times the standard deviation. Binding curve in panel A was carried out at $35^{\circ} \mathrm{C}$ in a $100 \mu \mathrm{L}$ solution obtained by mixing $10 \mu \mathrm{L}$ of blank blood plasma with $90 \mu \mathrm{L}$ of $\mathrm{Na}_{2} \mathrm{HPO}_{4}(50 \mathrm{mM})$ and $\mathrm{NaCl}(150$ $\mathrm{mM})$ at $\mathrm{pH} 7.0$ containing the AT20-reporter and input modules (strand \#1, $20 \mathrm{nM}$; strand \#2, $40 \mathrm{nM}$; strand \#3, $20 \mathrm{nM}$ ) and different concentrations of anti-AT2o antibodies. To detect the presence of anti-AT2o antibodies in blood plasma sample of HIV-infected patients, 90 $\mu \mathrm{L}$ of the above buffer solution containing the AT2o-reporter and the input module was added to $10 \mu \mathrm{L}$ of the blood plasma sample and the fluorescence signal measured after 10 minutes.

Our sensing strategy thus appears potentially useful for monitoring the immune response (i.e. anti-AT2o antibodies titers) of patients which have been subjected to AT2o-based HIV-vaccination. Specifically, we used a new PNA-peptide chimera as strand \#2 in which the specific AT2o peptide is employed as the recognition element. We first tested our platform using anti-AT2O targeting sensor antibodies. Such anti-AT2o monoclonal antibodies have been developed following immunization of Balb/c mice with AT2O and have been already used as positive controls in competition ELISA assays. ${ }^{26}$ Our platform is, once again, able to detect the target antibody in a rapid and highly sensitive fashion in a $10 \%$ blood plasma (see Figure 6a-b). Of note, titration of the antiHIV-1 p17 sensor with anti-AT20 monoclonal antibodies demonstrates that, as expected, the p17 antigen is not able to detect anti-AT2o antibodies (Figure SIn). We also note that the platform is quite stable over the time course of the experiment in 10\% plasma (Figure SI10). We then used our sensor to monitor the long-lasting immune response in HIV-1-infected patients enrolled in a medical trial and vaccinated with the synthetic AT2o peptide. As previously described, HIV-infected patients did not show consistent levels of anti-AT20 antibodies whereas $100 \%$ of immunized subjects developed high titers of AT20specific antibodies. ${ }^{27}$ We tested our sensor on samples collected by ten HIV-infected patients, seven of them vaccinated with AT2o-KLH. Fluorescence measurements demonstrate that our nucleic acid nanoswitch is able to detect the presence of anti-AT2o antibodies with high specificity in $10 \%$ human blood plasma solution of HIVinfected patients. Moreover, our single step fluorescence experiment allows to discriminate in less than ten minutes between HIV-infected patients which are under AT2o-based vaccine treatments and HIV-infected patients not involved in the treatments (Figure 6c). The same plasma samples were also tested using a control ELISA assay giving comparable results in terms of positive/negative discrimination (Figure SI12).

\section{CONCLUSION}

As in the case of ligand-induced dimerization of cellular receptors that trigger a downstream cascade, we have been inspired to design and develop a new, highly versatile sensing platform that allows detection of clinically relevant IgG and IgE antibodies in complex matrices such as blood serum and plasma in few minutes and without washing steps. We designed fluorescencebased DNA-PNA nanoswitches with low background in the absence of the target antibody. The presence of the target antibody induces an increase of the probes local concentration which ultimately triggers their hybridization. The fluorescence signal generated by the binding-induced conformational change of the DNA-PNA probes system has been used to quantify target antibodies concentration in complex biological matrices.

We demonstrated that our platform can efficiently detect four different antibodies with high sensitivity (low nanomolar levels), excellent specificity (no significant cross-reactivity has been observed) and rapid response time (less than $5 \mathrm{~min}$ ). Our experiments have shown that the platform is able to efficiently detect antibodies even in complex biological matrices, such as blood serum and blood plasma. We also demonstrated that our method can be used for the simultaneous analysis of multiple antibody targets in the same solution.

Finally, we proposed a clinically relevant application of our method monitoring the immune response of HIVpositive patients enrolled in a medical trial and treated with a peptide-based (AT2o peptide) phase-I therapeutic vaccine. Specifically, we were able to discriminate between AT2o-positive subjects (vaccinated patients) and controls (unvaccinated patients). Our nanoswitch-based platform can thus be conveniently used in AT2o Phase II/III clinical trials allowing an extensive and costeffective monitoring of serological response, whereas the classic ELISA/RIA approach might limit or restrict the analysis to fewer time-points due to costs and time that characterize these assays.

In conclusion we note that, while our direct system, as most of the approaches that do not rely on any amplification step, could not reach the sensitivity of ELISA/RIA methods, it presents features that make it 
highly advantageous in clinical applications. Apart from the rapid response and high specificity discussed above, the approach is also extremely versatile and can in principle be easily adapted to the detection of any antibody, antigen and bivalent macromolecular targets provided that the relevant recognition element can be coupled to a nucleic acid strand. The method also offers the possibility of multiplexing and, given that it is less reagent-intensive, is inexpensive. For example, a cost of about $0.01 \$$ /sample, which is approximately one order of magnitude lower than that of ELISA assays, can be estimated for the Anti-AT2o antibodies detection platform. The same sensing principle could also be adapted to other output signals (i.e. electrochemical or UV-VIS) thus making the platform even more suitable for Point-of-Care applications and large-scale analysis.

\section{EXPERIMENTAL SECTION}

Reagents and Materials. All chemicals were of analytical grade and were purchased from Sigma-Aldrich (St. Louis, MO) unless otherwise indicated. Antibodies were purchased from Roche Diagnostic Corporation, Germany (sheep polyclonal anti-digoxigenin anti-DIG, anti-digoxigenin Fab fragments), Sigma-Aldrich, USA (mouse monoclonal anti-2,4-dinitrophenol anti-DNP), Bio-Rad, UK (mouse polyclonal anti-Salmonella), Zeptometrix Corporation, USA (murine monoclonal antip17, anti-HIV antibody). Anti-AT2o monoclonal antibody (MBS-3) was produced as previously described. ${ }^{27}$ Polyclonal antibodies to the p17 AT20 epitope were produced by immunizing mice with KLH-conjugated AT2o peptide (AT20-KLH), as reported elsewhere. ${ }^{28}$ All the antibodies were aliquoted in $\mathrm{PBS}$ pH 7.4 and stored at $4^{\circ} \mathrm{C}$ for immediate use or at $-20^{\circ} \mathrm{C}$ for long-term storage. Bovine blood serum was purchased from Sigma Aldrich and human blood plasma samples were collected from HIV-positive patients vaccinated or not with AT2o-KLH. ${ }^{29}$

Oligonucleotides. All oligonucleotide employed in this work were synthesized, labeled, and purified (HPLC and reverse phase) by IBA GmBH (Göttingen, Germany) and used without further purification. Unless otherwise stated the labeled oligonucleotides were dissolved in Millipore water at a concentration of $1 \mathrm{mM}$ while the nonlabeled oligonucleotides were dissolved in the relevant buffer at a concentration of $100 \mu \mathrm{M}$. The final concentration of the oligonucleotides was confirmed using Tecan Infinite Mzoopro (Männedorf, Switzerland) through NanoQuant Plate $^{\text {TM }}$. The sequences of the oligonucleotides used were as follows. Strand \#1 of the sensing platform (DNA-based stem-loop) was terminally modified with AF488 (AlexaFluor488) or AF555 (AlexaFluor555) or AF68o (AlexaFluor680) and internally with BHQ-1 (black hole quencher 1) or BHQ-2 (black hole quencher 2) as noted below. Internal modifications were linked to these oligonucleotides via the $C_{5}$-position of the appropriate thymine base. PNA/Peptide chimera probes were purchased from Panagene, South Korea. The sequences and modification schemes of the oligonucleotide sequences are as follows.

\section{1) Anti-DNP targeting nanoswitches}

Strand \#1:

5'-(AF555)-T GAGCG GTA TAG CCT AAT TCG CGCTC T (BHQ2) CTG TCA CTT TCT GAG -3'

DNP-modified Strand \#2:

5'DNP-TTT TT CTC AGA AAG TGA CAG-3'

DNP-modified input strand (strand \#3) (16-mer):

5'DNP-TTT TTT TTT TTT TTT CGA ATT AGG CTA

TAC C-3'

DNP-modified input strand (strand \#3) (15-mer):

5'DNP-TTT TTT TTT TTT TTT CGA ATT AGG CTA TAC-3

DNP-modified input strand (strand \#3) (14-mer):

5'DNP-TTT TTT TTT TTT TTT CGA ATT AGG CTA TA-3'

DNP-modified input strand (strand \#3) (13-mer): $3^{\prime}$

5'DNP-TTT TTT TTT TTT TTT CGA ATT AGG CTA T-

2) Anti-DIG targeting nanoswitches

DIG Strand \#1:

5'-(AF488)-T AGG CG TTC TAG ATA TGC AAT CGC CT T (BHQ1) GTC TCA GAA TGT AAG-3'

DIG-modified Strand \#2:

5'DIG- TTT TT CTT ACA TTC TGA GAC-3'

DIG-modified input strand (strand \#3):

5'DIG-TTT TTT TTT TTT TTT ATT GCA TAT CTA GAA-3'

\section{3) Anti-p17 targeting nanoswitches}

p17 Strand \#1:

5'-(AF680)-T ACG TG ATC TAA TGG TGA GTC CAC GT T (BHQ2) CTA GAA TAA AAC GCC ACT G-3'

p17-modified Strand \#2 (PNA-Peptide chimera probe): $\mathrm{C}_{\text {term }}$

$\mathrm{N}_{\text {term }}$-ELDRWEKIRLRP-CAG TGG CGT TTT ATT CT-

p17-input strand (strand \#3):

5'AAG CTA TCC GAT CCA TTT TTT TTT TTT TTT TTT TT CAT TCG CTC CTT ACC-3'

\section{4) Anti-AT2o targeting nanoswitches}

AT2o Strand \#1:

5'-(AF680)-T ACG TG ATC TAA TGG TGA GTC CAC GT T (BHQ2) CTA GAA TAA AAC GCC ACT G-3'

AT2o-modified Strand \#2 (PNA-Peptide chimera probe):

$\mathrm{N}_{\text {term }}$-SGGELDRWEKIRLRPGGKKK-CAG TGG CGT TTT ATT- $\mathrm{C}_{\text {term }}$

AT2o-input strand (Strand \#3): 
5'AAG CTA TCC GAT CCA TTT TTT TTT TTT TTT TTT TT CAT TCG CTC CTT ACC-3'

In the above sequences the italic bases denote the poly-thymine tails and linkers introduced to enhance flexibility of the system. Underlined bases represent peptide portion (p17 and AT20 peptide) that are terminally conjugated to PNA (PNA-Peptide chimera). In bold are reported the five bases of the stem of the DNAbased nanoswitches.

Fluorescence experiments. All DNA oligos were stored $(100 \mu \mathrm{M})$ and used in a $100 \mathrm{mM}$ TRIS buffer, $\mathrm{pH} 8$. Fluorescence experiments were conducted at $\mathrm{pH} 7.0$ in 50 $\mathrm{mM} \mathrm{Na} \mathrm{HPO}_{4}$ buffer, $150 \mathrm{mM} \mathrm{NaCl}$ in a $100 \mu \mathrm{L}$ cuvette. Equilibrium fluorescence measurements were obtained using a Cary Eclipse Fluorimeter respectively with excitation at $480( \pm 5) \mathrm{nm}$ (for DNA nanoswitches labeled with FAM) and acquisition at $517( \pm 5) \mathrm{nm}$, with excitation at $555( \pm 5) \mathrm{nm}$ (for DNA nanoswitches labeled with AF555) and acquisition at $575 \mathrm{~nm}$ and with excitation at $680( \pm 5) \mathrm{nm}$ (for DNA-switches labeled with AF680) and acquisition at $702( \pm 5) \mathrm{nm}$. Binding curves reported in Figure 2 were obtained using $10 \mathrm{nM}$ of the DNP strand \#1 and DNP-modified strand \#2 by sequentially increasing the concentration of the DNP-modified input strand (strand $\#_{3}$ ) in the absence and presence of anti-DNP antibodies (i.e. $100 \mathrm{nM}$ ). Binding curves reported in Figure 3 were obtained using $10 \mathrm{nM}$ of the DNP strand \#1, DNP-modified strand \#2 and DNP-modified input strand and by sequentially increasing the concentration of antiDNP antibodies. Binding curves reported in Figure 4 and 6 were achieved using $20 \mathrm{nM}$ of p17/AT2o strand \#1 and p17/AT2o-input strand (strand \#3) in the presence of 40 $\mathrm{nM}$ of p17/AT2o-modified strand \#2. For each concentration the fluorescence signal was recorded every 10 minutes until it reached equilibrium. To monitor the immune response of patients under HIV-vaccine treatments, $90 \mu \mathrm{L}$ of $\mathrm{Na}_{2} \mathrm{HPO}_{4}(50 \mathrm{mM})$ and $\mathrm{NaCl}(150$ $\mathrm{mM}$ ) at $\mathrm{pH}$ 7.0 containing the AT20-reporter and input modules (strand \#1, $20 \mathrm{nM}$; strand \#2, $40 \mathrm{nM}$; strand \#3, $20 \mathrm{nM}$ ) were added to $10 \mu \mathrm{L}$ of blood plasma sample and the fluorescence signal measured after 10 minutes. Binding curves were obtained by plotting signal gain $\%$ (SG\%) vs concentration of target (i.e. antibody) in solution, and fitting the data with the following simplified Langmuir-type equation:

$$
\mathrm{I}_{\text {Fluo }}=\mathrm{a}+\mathrm{b} * \frac{[\text { Target }]}{\mathrm{K}_{1 / 2}+[\text { Target }]}
$$

Where $\mathrm{A}$ is the fluorescence background, $\mathrm{B}$ is the maximum signal gain $\left(S G \%_{\max }\right)$ and $K_{1 / 2}$ is the concentration of target at half-maximum signal gain. Signal Gain \% is calculated from the enhancement of fluorescence intensity achieved upon the addition of the antibody, relatively to the initial signal with the strand \#1, \#2 and \#3 mixture using the following formula:

$$
\% \text { Signal Gain }=\frac{\left[\mathrm{I}_{\text {Fluo }}(\mathrm{T})-\mathrm{I}_{\text {Fluo }}(0)\right]}{\mathrm{I}_{\text {Fluo }}(0)} * 100
$$

where $I_{\text {Fluo }}(T)$ is the fluorescence signal obtained in the presence of the target antibody and $\mathrm{I}_{\text {Fluo }}(\mathrm{o})$ is the fluorescence signal obtained in the absence of the target. To allow for more ready interpretation of the results, fluorescence signal obtained have been normalized on a o-1 scale using the following formula:

$$
\text { Rel. Fluor. }=\frac{\left[\mathrm{I}_{\text {Fluo }}(\mathrm{T})-\mathrm{I}_{\text {Fluo }}(0)\right]}{\mathrm{I}_{\text {Fluo }}(\mathrm{Max})-\mathrm{I}_{\text {Fluo }}(0)} * 100
$$

Where $I_{\text {Fluo }}(\max )$ represents the maximum fluorescence signal of the platform related to saturating concentration of the target (Rel. Fluor. $=1$ ) whereas $\mathrm{I}(\mathrm{o})$ is the background signal (Rel. Fluor. $=0$ ) and so the signal of the platform in the absence of the target.

ELISA assays. Anti-AT2o specific antibodies in plasma samples obtained from patients vaccinated or not with AT20-KLH were also detected using a conventional ELISA. ${ }^{29}$ Each well of 96-well microtiter plates (Maxisorp; Nunc, Roskilde, Denmark) was coated with $10 \mu \mathrm{g} / \mathrm{mL}$ of AT2o in $100 \mu \mathrm{L}$ of carbonate/bicarbonate buffer, $\mathrm{pH}$ 9.6, overnight at room temperature. Wells were then saturated with $200 \mu \mathrm{L} /$ well of PBS containing $2 \%$ bovine serum albumin (BSA) (wt/vol; assay buffer) for $1 \mathrm{~h}$ at $37^{\circ} \mathrm{C}$, and allowed to react with plasma diluted in assay buffer from 1:100 to 1:12.80o for $30 \mathrm{~min}$ at $37^{\circ} \mathrm{C}$. After washing 4 times with PBS containing $0.1 \%$ Tween-20 (vol/vol; washing buffer), the amount of bound antibody was determined by adding HRP-labeled goat anti-human or anti-mouse IgG.

\section{ASSOCIATED CONTENT}

Supporting Information. Binding curves, control experiments and specificity tests for anti-DIG, anti-DNP and anti-HIV 1 p17 antibodies detection, competitive fluorescence antibodybased detection of DNP in solution and comparison with ELISA method for Anti-AT20 antibodies detection are available. The Supporting Information is available free of charge on the ACS Publications website at http://pubs.acs.org.

\section{AUTHOR INFORMATION}

\section{Corresponding Authors}

*francesco.ricci@uniromaz.it

\section{Author Contributions}

\# These authors contributed equally. All authors have given approval to the final version of the manuscript.

Notes

The authors declare no competing financial interest. 


\section{ACKNOWLEDGMENT}

This work was in part supported by Associazione Italiana per la Ricerca sul Cancro, AIRC (project n. 14420) (FR).

\section{REFERENCES}

(1) Cutts, J. C.; Powell, R.; Agius, P. A.; Beeson, J. G.; Simpson, J. A.; Fowkes, F. J. I. BMC Med. 2014, 12, 150-170.

(2) Gubala, V.; Harris, L. F.; Ricco, A. J.; Tan, M. X.; Williams, D. E. Anal. Chem. 2012, 84, 487-515.

(3) Gao, W.; Emaminejad, S.; Nyein, H. Y. Y.; Challa, S.; Chen, K.; Peck, A.; Fahad, H. M.; Ota, H.; Shiraki, H.; Kiriya, D.; Lien, D.H.; Brooks, G. A.; Davis, R. W.; Javey, A. Nature 2016, 529, 509514 .

(4) Perfézou, M., Turner, A., Merkoçi, Chem. Soc. Rev., 2012, 41, 2606-2622.

(5) Nunes Pauli, G. E.; De La Escosura-Muñiz, A.; Parolo, C.; Helmuth Bechtold, I.; Merkoçi, A. Lab Chip 2017, 15, 399-405.

(6) Engelen, W.; Meijer, L. H. H.; Somers, B.; De Greef, T. F. A.; Merkx, M. Nat. Commun. 2o17, 8, 14473-14481.

(7) Janssen, B. M. G.; Van Rosmalen, M.; Van Beek, L.; Merkx, M. Angew. Chem. Int. Ed. 2015, 54, 2530-2533.

(8) Tian, L.; Heyduk, T. Anal. Chem. 2oo9, 81, 1147-1156.

(9) Heyduk, E.; Dummit, B.; Chang, Y.-H.; Heyduk, T. Anal. Chem. 2oo8, 8o, 5152-5159.

(10) Geddie, M. L.; Matsumura, I. J. Mol. Biol. 2007, 369, 10521059 .

(11) Banala, S.; Aper, S. J. A.; Schalk, W.; Merkx, M. ACS Chem. Biol. 2013, 8, 2127-2132.

(12) Arts, R.; Den Hartog, I.; Zijlema, S. E.; Thijssen, V.; Van Der Beelen, S. H. E.; Merkx, M. Anal. Chem. 2016, 88, 4525-4532.

(13) Mahshid, S. S.; Camiré, S.; Ricci, F.; Vallée-Bélisle, A. J. Am. Chem. Soc. 2015, 137, 15596-15599.
(14) White, R. J.; Kallewaard, H. M.; Hsieh, W.; Patterson, A. S.; Kasehagen, J. B.; Cash, K. J.; Uzawa, T.; Soh, H. T.; Plaxco, K. W. Anal. Chem. 2012, 84, 1098-1103.

(15) Vallée-Bélisle, A.; Ricci, F.; Uzawa, T.; Xia, F.;, Plaxco, K.W.J. Am. Chem. Soc. 2012, 134, 15197-15200.

(16) Plaxco, K. W.; Cash, K. J.; Ricci, F. J. Am. Chem. Soc., 2oo9, 131, 6955-6957.

(17) Lubin, A. A.; Plaxco, K. W. Acc. Chem. Res., 2010, 43, 496505 .

(18) Zhang, Z.; Hejesen, C.; Kjelstrup, M. B.; Birkedal, V.; Gothelf K. V. J. Am. Chem. Soc. 2014, 136, 11115-11120.

(19) Ranallo, S.; Rossetti, M.; Plaxco, K. W.; Vallée-Bélisle, A.; Ricci, F. Angew. Chem. Int. Ed. 2015, 54, 13214-13218.

(20) Lu, K.; Duan Q.P.; Ma, Li; Zhao D. Bioconjugate Chem. 2010, 21, 187-202.

(21) Trads, J.B.; Tørring, T.; Gothelf, K.V. Acc. Chem. Res. 2017, 50, 1367-1374.

(22) Rosier, B. J. H. M.; Cremers, G. A. O.; Engelen, W.; Merkx, M.; Brunsveld, L.; De Greef, T. F. A. Chem. Comm. 2017, 53, 73937396.

(23) Singhal, A.; Bagnacani, V.; Corradini, R.; Nielsen, P. E. ACS Chem. Biol.2014, 9, 2612-2620.

(24) De Gregorio, E.; Rappuoli, R. Nat. Rev. Immunol. 2014, 14, 505-514.

(25) Jones, L. H. Nat. Chem. 2015, 7, 952-960.

(26) Iaria, M. L.; Fiorentini, S.; Focà, E.; Zicari, S.; Giagulli, C.; Caccuri, F.; Francisci, D.; Di Perri, G.; Castelli, F.; Baldelli, F.; Caruso, A. Vaccine 2014, 32, 1072-1078.

(27) Fiorentini, S.; Marsico, S.; Becker, P. D.; Iaria, M. L.; Bruno, R.; Guzmán, C. A.; Caruso, A. Vaccine 2oo8, 26, 4758-4865.

(28) Fiorentini, S.; Giagulli, C.; Caccuri, F.; Magiera, A. K.; Caruso, A. Pharmacol. Ther. 2010, 128, 433-444.

(29) Foca, E.; Iaria, M. L.; Caccuri, F.; Fiorentini, S.; Motta, D.; Giagulli, C.; Castelli, F.; Caruso, A. HIV Clin. Trials 2015, 16, 157162 
TOC Graphic

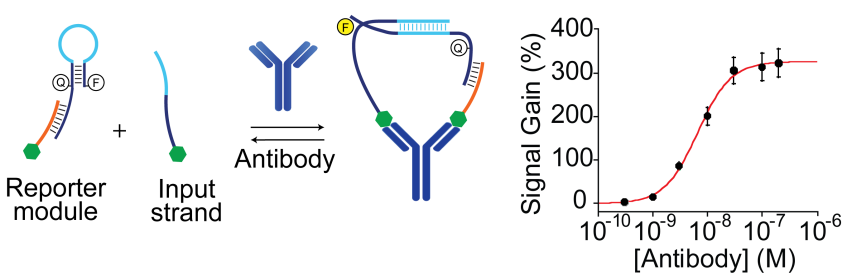

